\title{
Every Simplicial Polytope with at Most $d+4$ Vertices Is a Quotient of a Neighborly Polytope
}

\author{
U. H. Kortenkamp \\ Fachbereich Mathematik, Technische Universität Berlin, \\ Straße des 17. Juni 136, D-10623 Berlin, Germany \\ hund@math.tu-berlin.de
}

\begin{abstract}
We show that every simplicial $d$-polytope with $d+4$ vertices is a quotient of a neighborly $(2 d+4)$-polytope with $2 d+8$ vertices, using the technique of affine Gale diagrams. The result is extended to matroid polytopes.
\end{abstract}

\section{Introduction}

Neighborly polytopes are among the most interesting objects in polytope theory. The usual examples for neighborly polytopes are cyclic polytopes, first discovered by Carathéodory in 1904 . For a long time they were the only neighborly polytopes known in even dimensions, and one might ask if neighborliness is so restrictive that they are in fact the only ones. They are not, as has been shown by Grünbaum, and there are lots of them, as has been shown by Shemer [3]. In fact, it seems that the class of neighborly polytopes is so rich that it contains all simplicial polytopes as quotients.

Conjecture 1.1 (Perles/Sturmfels). Every simplicial polytope is a quotient of an (evendimensional) neighborly polytope. Similarly, every matroid polytope is a quotient (contraction) of a neighborly matroid polytope of odd rank.

Here we say that $P$ is a quotient of $P^{\prime}$ if its face lattice is an upper interval of the face lattice of $P^{\prime}$, that is, $P$ has the same combinatorial type as some face figure (or iterated vertex figure) of $P^{\prime}$.

A partial result was achieved by Sturmfels [4], who proved that the embedding may be done into an "almost neighborly" polytope. Here we prove Conjecture 1.1 for simplicial $d$-polytopes having at most $d+4$ vertices. 
Theorem 1.2. Every simplicial d-polytope with at most $d+4$ vertices is a quotient of a neighborly polytope of dimension at most $2 d+4$ and with at most $2 d+8$ vertices. Also, every matroid polytope of rank $d+1$ with at most $d+4$ vertices is a quotient of a neighborly matroid polytope of rank at most $2 d+5$ and with at most $2 d+8$ vertices.

\section{Gale Duality and Neighborly Polytopes}

The main tool used in this paper are Gale diagrams, which enable us to get a lowdimensional representation of high-dimensional polytopes with few (i.e., at most $d+4$ ) vertices. They were introduced by Gale, while Perles explored their full usability, as described in [2]. For the set-up of affine Gale diagrams, some applications and the detailed description of the connection between Gale diagrams and oriented matroid duality we refer to [4] and [5]. Here we just review some basic facts. Let us first recall the definition of a Gale transform.

Definition 2.1. Let $V=\left\{x_{1}, \ldots, x_{n}\right\}$ be a configuration of $n$ points affinely spanning $\mathbb{R}^{d}$. By embedding $V$ into the affine hyperplane $x^{(1)}=1$ of $\mathbb{R}^{d+1}$ we get a collection $\tilde{V}$ of $n$ vectors in $\mathbb{R}^{d+1}$. Then a configuration of $n$ vectors $V^{\prime}=\left\{x_{1}^{\prime}, \ldots, x_{n}^{\prime}\right\} \subset \mathbb{R}^{n-d-1}$ is a Gale transform of $V$ if its space of linear dependences is the orthogonal complement in $\mathbb{R}^{n}$ of the space of linear dependences of $\tilde{V}$.

One can reduce the dimension of the Gale transform by one, by scaling the vectors such that they lie in a common affine hyperplane (in general position).

Definition 2.2. The affine Gale diagram $V^{\prime}$ derived from a Gale transform $V^{\prime}=$ $\left\{x_{1}^{\prime}, \ldots, x_{n}^{\prime}\right\}$ in $\mathbb{R}^{n-d-1}$ is a set $\bar{V}=\left\{\bar{x}_{1}, \ldots, \bar{x}_{n}\right\} \subset H$ with $\bar{x}_{i}=\lambda_{i} x_{i}^{\prime}, \lambda_{i} \in \mathbb{R} \backslash\{0\}$, and $H$ is an affine hyperplane in general position of dimension $n-d-2$. Here $\bar{x}_{i}$ is called a black point if $\lambda_{i}>0$, and a white point otherwise.

The main point in Gale diagram theory is the fact that all combinatorial properties of a vector configuration may be read off the Gale transforms. In particular, we can draw (on a two-dimensional piece of paper) the affine Gale diagrams of polytopes of arbitrary dimension $d$, provided they have at most $d+4$ vertices. The connection between the oriented matroid of a vector configuration and its Gale transform (see Chapter 9 of [1] and Chapter 6 of [6] for detailed discussions) is given by the following theorem.

Theorem 2.3. The oriented matroids of a vector configuration $V$ and its Gale transform $V^{\prime}$ are dual to each other: $\mathcal{M}(V)^{*}=\mathcal{M}\left(V^{\prime}\right)$. In particular, the cocircuits of $V$ are the circuits of $V^{\prime}$, and vice versa.

Proof. See Corollary 6.15 of [6].

In particular, this implies that the quotients of a polytope are obtained by deletion of points from its affine Gale diagram. Now we use this for studying neighborly polytopes and their quotients. 
Definition 2.4. A $d$-polytope $P$ is $k$-neighborly if any $k$ vertices form the vertex set of a face. A $d$-polytope $P$ is neighborly if any set of $\lfloor d / 2\rfloor$ vertices forms the vertex set of a face of $P$.

The fact that the simplex is the only $d$-polytope which is $k$-neighborly for $k>\lfloor d / 2\rfloor$ gives rise to the above definition. The neighborliness condition is very weak in odd dimensions, see the case $d=3$. Hence we restrict ourselves-as is customary in the theory of neighborly polytopes - to even dimensions.

The combinatorial property of Gale transforms corresponding to neighborliness is as follows.

Definition 2.5. A $d$-dimensional vector configuration $V$ on $n=d-1+2 k$ vectors is balanced if for every hyperplane spanned by $d-1$ vectors of $V$ there are exactly $k$ vectors on either side of the hyperplane.

Remark 2.6. Definition 2.5 implies that the vectors of $V$ are in general position. Also observe that the dimension of the dual configuration is even.

When using affine $(d-1)$-dimensional Gale diagrams, it is easy to check balancedness of the associated vector configuration. Any $d-1$ points have to span a hyperplane, and there has to be the same number of points on either side, where we count black points as positive and white points as negative.

As an example for balanced vector configurations we like to mention the Gale transforms of even-dimensional cyclic polytopes. But in fact one can characterize all the balanced Gale transforms by the following lemma.

Lemma 2.7. The Gale transforms of simplicial neighborly $2 d$-polytopes with $n=$ $2 d+k$ vertices are exactly the balanced $(k-1)$-dimensional configurations of $n$ vectors.

Proof. We first show that any balanced vector configuration $V$ is the Gale transform of a neighborly polytope. Observe that any cocircuit has exactly $d+1 \geq 2$ positive elements (and the same number of negative elements) due to the balancedness, thus the vector configuration is indeed the Gale transform of a convex polytope (see Theorem 6.19 of [6]), which is simplicial because of Remark 2.6. This polytope is neighborly if any $d$ vectors define a face, which is the case if the remaining $n-d=d+k$ vectors are positively dependent in the Gale transform. By the Farkas lemma (as stated in Proposition 1.10 of [6]) we know that the remaining vectors are either positively dependent or form a cone $\mathcal{C}$ which is not the whole $\mathbb{R}^{k-1}$. In the second case, we look at a hyperplane spanned by a facet $\mathcal{F}$ of $\mathcal{C}$. All of the remaining vectors lie in the same closed half-space which is defined by $\mathcal{F}$. But this is a contradiction to the balancedness of $V$, since we deleted less than $d+1$ vectors from our configuration, so there has to remain at least one point on either side.

Now suppose we are given any simplicial neighborly $2 d$-polytope with $2 d+k$ vertices, which implies that its vertices are in general position, see [3]. So we know that any $d+k$ vectors in its Gale transform are positively dependent and in general position. If the Gale 
transform were not balanced, there would be a set of more than $d+1$ points lying on one side of a hyperplane through $k-2$ points, thus there would be at least $d+k$ points which are not positively dependent, which contradicts our assumption.

\section{Embedding Oriented Matroids}

Since we are just dealing with the combinatorial properties of Gale transforms, it is convenient to look at their oriented matroids. The following definition is compatible to Definition 2.5, in the sense that the oriented matroid of a vector configuration is balanced if and only if the vector configuration itself is balanced.

Definition 3.1. A rank- $(d+1)$ oriented matroid on $k$ elements is balanced (or dual neighborly) if and only if $k-d$ is even and every cocircuit has exactly $(k-d) / 2$ positive and the same number of negative elements.

Given that definition, one can reformulate Lemma 2.7 to be valid for matroid polytopes also, which is proven quite literally.

Lemma 3.2. The dual of any neighborly matroid polytope of rank $2 d+1$ with $n$ vertices is a balanced oriented matroid on n elements of rank $n-2 d-1$, and vice versa.

Now we are prepared for the key theorem of this paper, which implies Theorem 1.2.

Theorem 3.3. Any uniform rank-3 oriented matroid $\mathcal{M}$ on $k$ elements may be embedded into a balanced rank-3 oriented matroid $\mathcal{M}^{\prime}$ on $2 k$ elements.

Proof. We give a construction sequence that describes the embedding, using the construction methods as described in Section 7.2 of [1]. Let $\mathcal{M}$ be a rank-3 oriented matroid on the set $E=\left\{e_{1}, \ldots, e_{k}\right\}$. Start with a lexicographic extension ${ }^{1}$ by an element $\overline{\bar{e}}_{1}:=\left[e_{1}^{+} e_{2}^{+} e_{3}^{+}\right]$, obtaining $\mathcal{M}^{(1)}=\mathcal{M}\left[e_{1}^{+} e_{2}^{+} e_{3}^{+}\right]$which has the contravariant pair $\left\{e_{1}, \overline{\bar{e}}_{1}\right\}$. Now we perform a lexicographic extension on each of the remaining elements: For every $e_{i}, 2 \leq i \leq k$, extend $\mathcal{M}^{(i-1)}$ by $\bar{e}_{i}:=\left[e_{i}^{-} e_{1}^{-}\left(\overline{\bar{e}}_{1}\right)^{-}\right]$obtaining $\mathcal{M}^{(i)}$. Every step in our construction gives one covariant pair $\left\{e_{i}, \bar{e}_{i}\right\}, 2 \leq i \leq k$, which is not destroyed by the subsequent operations, because no point is involved twice, in contrast to the contravariant pair in the first step, which does not exist anymore. The resulting oriented matroid $\mathcal{M}^{\prime}:=\mathcal{M}^{(k)}$ is the one we are looking for. It is uniform because all lexicographic extensions were done on complete bases.

We have to show that $\mathcal{M}^{\prime}=\mathcal{M}^{\prime}\left(E \cup\left\{\overline{\bar{e}}_{1}, \bar{e}_{2}, \ldots, \bar{e}_{k}\right\}\right)$ is dual neighborly. There are four main cases to check: the cocircuit defined by $e_{1}$ and $\overline{\bar{e}}_{1}$, cocircuits defined by $e_{i}$ and

\footnotetext{
${ }^{1}$ For the reader who is not familiar with lexicographic extensions we wish to explain them in the realizable rank-3 case. Let $\mathcal{M}$ be the oriented matroid of a vector configuration $V=\left\{x_{1}, \ldots, x_{n}\right\}$, and choose $\alpha, \beta, \gamma \in$ $\{+1,0,-1\}$ and $i, j, l \in\{1, \ldots, n\}$. Then we extend $V$ by a new vector $\bar{x}=\alpha x_{i}+\varepsilon \beta x_{j}+\varepsilon^{2} \gamma x_{l}$ with $\varepsilon>0$ being small, which is a vector very close to $\alpha x_{i}$ and slightly displaced in the directions of $\beta x_{j}$ and $\gamma x_{l}$. The oriented matroid of $V \cup\{\bar{x}\}$ is the lexicographic extension $\mathcal{M}\left[i^{\alpha}, j^{\beta}, l^{\gamma}\right]$ of $\mathcal{M}$.
} 


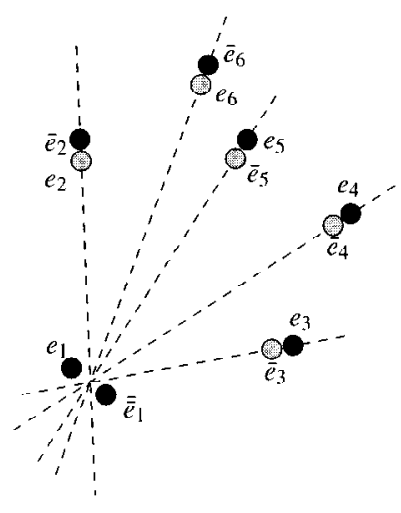

(a)

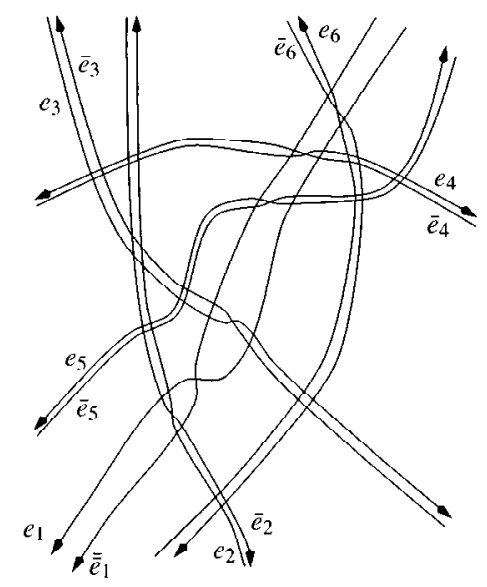

(b)

Fig. 1. The construction of the balanced oriented matroid as described in the proof of Theorem 3.3. (a) shows the realizable case, whereas (b) shows the general situation.

$\bar{e}_{i}$ for $2 \leq i \leq k$, cocircuits defined by $e_{i}$ or $\bar{e}_{i}$ and $e_{j}$ or $\bar{e}_{j}$ for $2 \leq i<j \leq k$ (mixed cocircuits), and cocircuits defined by $e_{1}$ or $\overline{\bar{e}}_{1}$ and $e_{i}$ or $\bar{e}_{i}$ for $2 \leq i \leq k$ (completely mixed cocircuits) (see Fig. 1(a)).

In the first case we have $k-1$ inseparable pairs of covariant elements by construction, none being dependent on $e_{1}$ or $\overline{\bar{e}}_{1}$, so we have $k-1=(2 k-2) / 2$ positive and $k-1$ negative signs as desired. The second case is easy as well. The $k-2$ remaining covariant pairs are contributing $k-2$ sign pairs, and $e_{1}$ and $\overline{\bar{e}}_{1}$ have different signs with respect to $e_{i}$ and $\bar{e}_{i}$ by construction of $\bar{e}_{i}$, thus giving the missing sign pair.

The mixed cocircuits are a little bit more tricky to handle. First, we get $k-3$ covariant pairs of elements $\left\{e_{l}, \bar{e}_{l}\right\}$ for $l \neq i, j$. But now $e_{1}$ and $\overline{\bar{e}}_{1}$ amount to the same sign, because they were constructed as contravariant elements with respect to $E$, and since we did extend the matroid by lexicographic extensions involving at most one of the $e_{l_{1}}$ and $e_{l_{2}}$, $2 \leq l_{1}, l_{2} \leq k$, we get the same orientation of $e_{1}$ regardless of using $e_{i}$ or $\bar{e}_{i}$. These two signs get compensated for by the remaining two elements from our pairs $\left\{e_{i}, \bar{e}_{i}\right\}$ and $\left\{e_{j}, \bar{e}_{j}\right\}$, thus completing the two remaining sign pairs.

Finally, we look at the completely mixed cocircuits. The situation is similar to the one before. We have $k-2$ covariant pairs of elements. But now the remaining point $\tilde{e}_{1}$ of $\left\{e_{1}, \overline{\bar{e}}_{1}\right\}$ is either on the same side as the remaining point $\tilde{e}_{i}$ of $\left\{e_{i}, \bar{e}_{i}\right\}$ or on the other one. Luckily, we chose the direction of construction of $\bar{e}_{i}$ such that the point having the same orientation as $e_{1}$ and $\overline{\bar{e}}_{1}$ is on the opposite side of $\tilde{e}_{1}$, and the other one, having the other orientation, is on the same side as $\tilde{e}_{i}$ (with regard to the hyperplane spanned by the remaining two points). This completes our proof.

Corollary 3.4. Every uniform matroid polytope of rank $d+1$ with at most $d+4$ vertices is a quotient of a uniform neighborly matroid polytope of rank at most $2 d+1$ with at most twice as many vertices. 
Proof. The cases of matroid polytopes having less than $d+3$ vertices are trivial. If the matroid polytope has exactly $d+3$ vertices then its dual is a rank- 2 uniform oriented matroid on $d+3$ elements, i.e., a number of black and white points on the line. Adding at most $d$ additional points between adjacent points of the same color gives us an alternating sequence of black and white points of odd length. This sequence is balanced and we are done by Lemma 3.2. Given any matroid polytope $\mathcal{M}$ of rank $d+1$ having $n=d+4$ vertices, look at its dual oriented matroid $\mathcal{M}^{*}$. This may be embedded into a balanced oriented matroid $\mathcal{M}^{* \prime}$ having $2 n$ vertices by Theorem 3.3, whose dual $\mathcal{M}^{* * *}$ is neighborly by Lemma 3.2. Since we obtain the original $\mathcal{M}$ oriented matroid by deletion of elements of $\mathcal{M}^{* * *}$, we found $\mathcal{M}$ to be a quotient of $\mathcal{M}^{* * *}$.

Finally, we complete the proof of Theorem 1.2.

Proof. We may assume that the vertices of the simplicial $d$-polytope are in general position. So the theorem follows from Corollary 3.4 and the fact that a series of lexicographic extensions does not spoil realizability.

\section{Embedding (Pseudo-)Line Arrangements}

The above construction is based on a much simpler task. We describe the idea behind it in order to provide a possible starting point for further investigation.

Definition 4.1. Let $\mathcal{A}$ be a (pseudo-)line arrangement in the projective plane. A cell in the arrangement is even if it has an even number of sides, odd otherwise. An arrangement $\mathcal{A}$ is good if it has a two-coloring of the cells with adjacent cells colored differently and all odd cells having the same color.

A good arrangement has an even number of lines, since otherwise it could not be two-colored. Also, it is not possible for an arrangement to have only even cells, since any planar (pseudoline-)arrangement has at least one triangle, i.e., an odd cell. However, we have the following:

Theorem 4.2. Every planar (pseudo-)line arrangement $\mathcal{A}$ may be embedded into a planar (pseudo-)line arrangement $\mathcal{A}$ which is good.

Proof. The proof is essentially the same as the one of Theorem 2.7, omitting the orientations. In fact, we are creating an arrangement which may be colored in such a way that one color is covering only cells having four or six edges (or vertices). These are the ones that are between two co- (resp. contravariant) elements (like $e_{i}$ and $\bar{e}_{i}$, see Fig. 1(b)). The cells of the original arrangement can be found again as the cells which are adjacent to at most one line of each pair $\left\{e_{1}, \overline{\bar{e}}_{1}\right\}$ and $\left\{e_{i}, \bar{e}_{i}\right\}$.

The good thing about good arrangements is that they may be given orientations such that they are balanced. Here we mean by balanced that its oriented matroid is balanced, which gives: 
Definition 4.3. An arrangement $\mathcal{A}$ of oriented (pseudo-)lines in the (Euclidean) plane is balanced, if any crossing of two lines lies to the right of one half of the other lines and to the left of the other half. An arrangement $\mathcal{A}$ of oriented (pseudo-)lines in the projective plane is balanced, if we can fix an additional projective line $\ell$ in general position such that the Euclidean arrangement we get by deleting $\ell$ from the projective plane is balanced.

Theorem 4.4. Every good arrangement may be oriented in a way that it is balanced.

Proof. We are given a two-colored arrangement with all cells of white color being even. Start by fixing the orientation of one line $\ell$ arbitrarily. Then the other lines have a fixed orientation by the rule that the orientations along the borders of the white cells adjacent to $\ell$ should alternate. This is well defined, it cannot happen that a line is given two different orientations. Suppose a line $\ell_{1}$ other than $\ell$ forms a border segment of two white cells $\mathcal{C}_{1}$ and $\mathcal{C}_{2}$ adjacent to $\ell$. The number of border segments from $\ell_{1}$ to $\ell$ on $\mathcal{C}_{1}$ added to the number of border segments from $\ell$ to $\ell_{1}$ on $\mathcal{C}_{2}$ is the number of lines crossing $\ell$ between $\mathcal{C}_{1}$ and $\mathcal{C}_{2}$. If $\mathcal{C}_{1}$ and $\mathcal{C}_{2}$ are on the same side of $\ell$ this number is even, giving the same orientation of $\ell_{1}$ both times. If they are on opposite sides, then $\ell$ is also assigned the same orientation both times, since the first segment of $C_{2}$ has the same orientation as the last of $\mathcal{C}_{1}$.

Now every white cell has the property that the lines on the border are alternatingly oriented, which will enable us to take any line as the special line $\ell$ : Consider the triangle formed by $\ell$ and two border lines of a white cell and all lines crossing this triangle, and check whether an even or an odd number of lines crosses at every side of the triangle. The total number of crossings has to be even (every line going in has to come out again). By distinguishing all the possible cases we are done.

The oriented arrangement we get is balanced. Take any crossing of two lines, one of them being without loss of generality $\ell$. Then the remaining lines cross $\ell$ alternatingly, and we have an even number of them, thus the crossing lies to the right of half the remaining lines and to the left of the other half.

There is a close relationship of the above to the problem of embedding polytopes into neighborly polytopes, although we start with an already oriented arrangement in the second case. Observe that the balanced arrangement corresponding to the neighborly polytope constructed in Section 3 is also good (but due to the given orientations we cannot use Theorem 4.2 to assign the orientations).

Problem 4.5. Find a good definition of good as in Definition 4.1 which serves for higher dimensions.

\section{Acknowledgments}

I am indebted to Günter Ziegler, who made me aware of the problem of line embeddings and their applications, to Jürgen Richter-Gebert for fruitful discussions, and to Bernd Sturmfels for initiating the discussion. 


\section{References}

1. A. Björner, M. Las Vergnas, B. Sturmfels, N. White, and G. M. Ziegler, Oriented Matroids, Cambridge University Press, Cambridge, 1993.

2. B. Grünbaum, Convex Polytopes, Interscience, London, 1967.

3. I. Shemer, Neighborly polytopes, Israel J. Math. 43 (1982), 291-314.

4. B. Sturmfels, Neighborly polytopes and oriented matroids, European J. Combin. 9 (1988), 537-546.

5. B. Sturmfels, Some applications of affine Gale diagrams to polytopes with few vertices, SIAM J. Discrete Math. 1 (1988), 121-133.

6. G. M. Ziegler, Lectures on Polytopes, Graduate Texts in Mathematics, vol. 152, Springer-Verlag, New York, 1995.

Received September 27, 1995. 